\title{
Labyrinthe
}

$24 \mid 2006$ (2)

Faut-il être postcolonial?

\section{The Location of Postcolonial Studies}

\section{Marc Aymes}

\section{OpenEdition}

Journals

Édition électronique

URL : http://journals.openedition.org/labyrinthe/1188

DOI : $10.4000 /$ labyrinthe. 1188

ISSN : 1950-6031

Éditeur

Hermann

Édition imprimée

Date de publication : 15 juin 2006

Pagination : 39-45

ISBN : 2-9526131-1-7

\section{Référence électronique}

Marc Aymes, "The Location of Postcolonial Studies », Labyrinthe [En ligne], 24 | 2006 (2), mis en ligne le 07 juin 2006, consulté le 06 mai 2019. URL : http://journals.openedition.org/labyrinthe/1188 ; DOI : 10.4000/labyrinthe.1188

Ce document a été généré automatiquement le 6 mai 2019.

Propriété intellectuelle 


\title{
The Location of Postcolonial Studies
}

\author{
Marc Aymes
}

Pour des raisons qui seront vite évidentes au

lecteur, ce texte est mis en ligne dans son intégralité dès la parution du numéro papier. Une

archive de chaque document cité ci-après est annexée au présent texte en version électronique ; de la sorte, tous resteront accessibles quel que soit le sort réservé aux originaux.

$1 \quad$ Localiser les études postcoloniales ? Ce qui suit pourtant n'est pas un atlas, relevé des plus massifs modelés et des plus infimes érosions affectant le continent du postcolonialisme. Ce n'est pas non plus un essai, articulant un corpus de propositions et de déductions. Il s'agirait plutôt d'un journal de terrain, pour emprunter (sauf leur respect) au vocabulaire des ethnologues. C'est-à-dire une suite de visions saisies sur le vif, d'impressions griffonnées avant qu'elles ne s'effacent. Le compte rendu d'une exploration assez méthodique pour être révélatrice, mais suffisamment disparate aussi pour ne pas être interminable.

2 Le séminaire-matrice de ce journal commandait une perspective de travail principalement transatlantique. Pour cette raison, mes sondages adoptent l'Amérique du Nord, les États-Unis surtout, comme domaine privilégié. Et ils ciblent délibérément une poignée d'universités de recherche, laissant de côté une myriade de colleges.

3 J'en viens à l'essentiel, cependant : l'enquête proposée ici a pour seuls fils conducteurs les liens hypertexte de l'internet ${ }^{1}$. Et du coup, elle n'adhère pas exactement aux territoires institutionnels et géographiques qu'elle parcourt. Elle a même tôt fait d'en passer les bornes. C'est pourquoi aussi on ne trouvera en définitive ici nulle cartographie précise du postcolonialisme dans l'Université américaine - mais plutôt un chapelet de situations énonciatives, qu'il semblait malgré tout possible de considérer, un temps, comme typiques.

4 Il y a, bien sûr, du postcolonial là-dedans. Quelque chose comme la recherche d'une «localité de la culture» des études postcoloniales elles-mêmes. Cette localité, pour emprunter à Homi Bhabha, serait 
une forme de vie plus complexe que la "communauté"; plus symbolique que la "société"; plus chargée de connotations que le "pays"; moins patriotique que la "patrie"; plus rhétorique que la raison d'État; plus mythologique que l'idéologie ; moins centrée que le citoyen; plus collective que "le sujet"; plus psychique que la civilité ; plus hybride dans l'articulation des différences et des identifications culturelles que ne peut le représenter une quelconque structuration hiérarchique ou binaire de l'antagonisme social ${ }^{2}$.

5 En ce sens (ou ce perpétuel non-sens, je ne sais), localiser les études postcoloniales devient une expérience $d$ '«interdisciplinarité », en tant qu'ouverture d'un "espace supplémentaire pour l'articulation de savoirs culturels adjacents et adjoints mais non nécessairement cumulatifs, téléologiques ou dialectiques ${ }^{3}$ ». C'est à ce titre que la divagation internautique peut apparaître comme une idée ad hoc.

6 Reste le péril, et il est grand. Volatil, proliférant, l'internet n'autorise souvent qu'un " butinage $e^{4}$ », et rarement une interprétation compréhensive des signaux qu'il charrie. On s'en tient, en somme, à l'observation d'artéfacts.

7 Cette conscience du danger ne vaut pas uniquement en ce qu'elle impose de s'entourer de précautions. Elle vaut aussi pour elle-même, en tant que mise en danger volontaire de nos topographies scientifiques acquises, de nos petites patries et de nos prés carrés. L'approche mérite donc d'être tentée parce que précisément elle ne peut être maitrisée.

Mots-clés

$8 \quad$ Plutôt que les parentés confessées en bas de page, nous explorons ici les mots-clés jetés en pâture aux moteurs de recherche.

9 Ils sont révélateurs à plus d'un titre. D'abord parce qu'ils mettent en évidence les influences d'un vocable, les modes affectant la manière dont les chercheurs se présentent à eux-mêmes et aux autres. Plus avant, ils révèlent aussi combien le travail de la recherche, dans une discipline donnée ou au-delà, suppose de choix stylistiques; et dans quelle mesure ceux-ci ont à voir avec des décisions théoriques, plus ou moins mûrement réfléchies.

10 Les mots-clés sont autant de chausse-trappes, cependant. Car le vagabondage hypertexte exclut de jamais prendre la mesure des distances séparant signifiants et signifiés :

11 1. Certains annoncent du postcolonial, mais rien ne permet de déceler autre chose qu'une simple commodité. Ainsi, je m'interroge sur l'usage de la notion parmi les historiens : le postcolonial, pour ceux d'entre eux qui y font référence, s'agit-il d'autre chose que d'un post-colonial (avec trait d'union)? Autrement dit, d'un simple après-colonial ? Et est-il vraiment question, par-delà le simple outil de périodisation, d'amorcer quelque délocalisation conceptuelle que ce soit ${ }^{5}$ ?

12 2. D'autres au contraire, bien que ne conférant aucune prééminence au terme, situent manifestement leurs travaux sur un terrain d'entente avec les postcolonial studies. Je note en particulier combien les anthropologues qui invoquent le registre postcolonial ne manquent jamais de le passer au crible des autres références conceptuelles disponibles dans la discipline. Aussi par exemple, dans l'auto présentation proposée par Maxine Weisgrau (Columbia, Anthropologie) ${ }^{6}$, la proposition d'une analyse des «discours coloniaux» va-t-elle de pair avec la reconduction de notions consacrées du questionnement anthropologique : «tribu », « rituel »...

Collocations 
13 Il apparait alors que le syntagme postcolonial est de toutes parts recoupé, débordé par d'insistants voisins.

14 1. Parmi eux figure le couple imperial / postimperial. Et voici que redouble l'ambiguïté notée ci-dessus : le soupçon s'insinue, à nouveau, que ce post- ne soit pas davantage qu'un après?

15 2. Une autre manière de dire (ou de suggérer) un rattachement au postcolonial est de faire référence au fondateur subaltern. Reste à savoir si cela revient toujours au même. On remarque que les principaux membres de la collection Subaltern Studies ne sont pas tous devenus figures de proue des études postcoloniales. Revendiquée par Dipesh Chakrabarty (Chicago, Histoire) ou Gayatri C. Spivak (Columbia, Littérature anglaise et comparée), une telle continuité semble simplement sous-entendue par Partha Chatterjee (Columbia, Anthropologie), et pour le moins introuvable dans le cas de Gyanendra Pandey (Emory, Histoire).

16 3. Il faut également compter avec certains nouveaux-venus, posés en synonymes de postcolonial. J'ai retenu l'un d'entre eux, exemplaire des dynamiques énonciatives dont le verbe postcolonial est traversé : transnational.

17 On remarque l'aisance avec laquelle ce mot côtoie le questionnement postcolonial. Michael Dietler (Chicago, Anthropologie et Études classiques) peut ainsi déclarer simultanément, d'une part, "mener des recherches archéologiques sur les sociétés "celtes" d'Europe à l'Âge du fer, notamment sur la rencontre coloniale avec les Étrusques, les Grecs et les Romains en France méridionale»; et, d'autre part, "explorer les politiques de la pratique archéologique et de l'usage du passé ancien en rapport avec la construction d'une identité et d'une mémoire sociale celtes, en contexte aussi bien moderne et ethno-nationaliste que post-moderne et transnational ».

18 Non loin de là, Prasenjit Duara (Chicago, Histoire) se donne les spécialités suivantes: " Histoire sociale et culturelle de la Chine moderne ; Nationalisme et transnationalisme ; Histoire et théorie post-structuraliste ». Tout en rappelant, à titre de confirmation de ses liens avec les études postcoloniales, qu'il est l'auteur de l'article " Postcolonial History » publié dans un Companion to Western Historical Thought ${ }^{8}$.

19 Plus à l'ouest, mais sur un terrain de recherches proche, Shu-mei Shih (Université de Californie à Los Angeles [= UCLA], Littérature comparée, Langues et cultures asiatiques et Asian American Studies) étudie « la littérature comparée, avec une spécialité et un intérêt pour les littératures chinoise, sinophones et Asian American. Ses thèmes de recherches comprennent également le féminisme transnational, une approche comparée du discours de minorité, le modernisme, le (post)humanisme, et le (post)colonialisme ». À nouveau mis en danger par des parenthèses qui en font vaciller la nécessité, le post- semble devoir s'accommoder d'un trans- qui sonne plus ambitieux que lui.

Succession

20 De fait, la collocation se mue bientôt en lutte de succession. Entre postcolonial et transnational, il faut choisir.

21 Cette substitution résolue d'un terme à l'autre est manifeste parmi certains anthropologues, dont le profil traduit le choix d'un transnational omniprésent : c'est le cas par exemple, à Columbia, de Nicholas De Genova, Brian Larkin, Rashmi Sadana et Sandhya R. Shukla ; et à Yale, de Kamari M. Clarke et Patricia R. Pessar9 . 
22 Soit aussi la revue Public Culture. Je perçois, dans les mots-clés qui marquent sa ligne éditoriale, les signes d'une même tendance. On remarque immédiatement que la revue est publiée par la Society for Transnational Cultural Studies (Duke University Press). On le remarque d'autant plus que le nom de cette dernière fait office de sous-titre du journal. En revanche, et bien que Dipesh Chakrabarty compte parmi les membres-pivots du comité éditorial, son argumentaire se dispense de toute référence directe au postcolonialisme.

23 Encore Public Culture a-t-elle la tempérance des revues installées, devenues incontournables. D’autres, plus récemment fondées, affirment plus sèchement la rupture avec le postcolonial - non sans recourir au stratagème d'un titre qui en porte les couleurs. Fondée en 2003 au Canada, la revue Postcolonial Text est la «publication officielle " de l'Association for Commonwealth Literature and Language Studies. Son nom de baptême dissimule des thèses autrement schismatiques :

Postcolonial Text encourage les débats critiques sur les études postcoloniales, ce terrain culturellement disputé et parfois théoriquement glissant. En particulier, cette revue en ligne examine la relation entre les études postcoloniales, les diaspora studies et de nouveaux champs en cours d'émergence tels les transnational cultural and globalization studies.

Dans le même temps, la revue reconnaît que les études postcoloniales peuvent être utilisées en tant que discours-maître de l'identité culturelle, tendant à homogénéiser et réguler des textes et des identités culturellement et géographiquement très différents. Afin d'entretenir la critique des formes instituées de la production du savoir culturel, Postcolonial Text s'engage à une analyse rigoureuse des relations de pouvoir néo-coloniales inégales entre le Nord et le Sud, au croisement de la classe, du genre et de la race.

24 La ligne éditoriale ainsi adoptée marque le souci de trouver un successeur au trop "glissant» postcolonial. Au même titre que transnational, neocolonial figure parmi les prétendants. C'est bien l'approche du colonial lui-même qu'il s'agit, à la faveur d'un nouveau préfixe, de réviser.

Reprenons et poursuivons alors le parcours de Shu-mei Shih, en tâchant de mieux discerner les liens et les structures qui l'informent. Son institution de rattachement, UCLA, est un lieu où l'orientation postcoloniale semble implantée sans réserves: un « champ de recherches » ne lui est-il pas réservé au département d'Études françaises et francophones («Francophone and Postcolonial Studies»), autour de Françoise Lionnet et Dominic Thomas - et de même au département d'Études anglaises ("Postcolonial and Anglophone Literature ») ? Par-delà les départements, et par-delà le seul campus de Los Angeles, une autre structure est là cependant, dont Shu-mei Shih assure (avec Françoise Lionnet) la direction. Elle est présentée comme une « communauté interdisciplinaire des chercheurs en sciences humaines et sociales de l'ensemble de l'Université de Californie ». Or ni son nom - le Transnational and Transcolonial Studies Multicampus Research Group - ni son objectif affirmé - «collaborer pour l'étude du discours de minorité par-delà les frontières nationales (transnational), en prêtant attention aux processus coloniaux et néo-coloniaux (transcolonial)» - ne reconduisent le vocable postcolonial. Mieux : il est souligné que «si à ce jour, le paradigme postcolonial est le seul disponible pour structurer l'étude des cultures coloniales et postcoloniales, il est insuffisant à bien des égards ».

Transcolonial: l'avènement de ce nouveau mot-clé, aboutissement d'un détour par le vocable du transnational, peut être lu comme un dénouement. Semblable tentative de 
refondation manifeste clairement que décidément le post- (trop ambigu, trop diachronique) ne convenait plus. Lui substituer le trans-, ce n'est pas simplement l'investir de nouvelles ressources thématiques (telles celles que suggèrent les diaspora studies mentionnées plus haut) : c'est bel et bien tenter de mettre en place un nouveau référent herméneutique.

\section{NOTES}

1. Consultation effectuée en mars 2006. Une archive de chaque document cité ci-après est annexée au présent texte en version électronique ; de la sorte, tous resteront accessibles quel que soit le sort réservé aux originaux.

2. The Location of culture, Londres/New York, Routledge, 1994, p. 140 (je traduis, ici comme ci-après).

3. Ibidem, p. 163.

4. Traduction possible de l'anglais browsing.

5. Voir ainsi le profil de Gregory Mann (Columbia, Histoire). Sur cette question, voir aussi le texte d'Anthony Mangeon dans ce numéro.

6. Voir aussi une autre présentation de la même personne, où la reformulation inclut cette fois explicitement la référence postcoloniale.

7. Ainsi le projet Postcolonial Web, hébergé par l'Université nationale de Singapour et animé par George P. Landow (Brown), propose un panorama de la « Contemporary Postcolonial and Postimperial Literature in English ».

8. Publié sous la direction de Sarah Maza et Lloyd Kramer ; Malden (Mass.), Blackwell, 2002.

9. En co-affiliation avec le programme d'Études américaines pour cette dernière. 